\title{
Acetic acid production by selective oxidation of ethanol using Au catalysts supported on various metal oxide
}

\author{
Steton M. Tembe ${ }^{1}$, Gary Patrick², Mike S. Scurrell ${ }^{1, *}$ \\ www.goldbulletin.org
}

\begin{abstract}
The liquid-phase oxidation of ethanol to acetic acid using $\mathrm{Au}$ catalysts supported on various metal oxides was studied at $150^{\circ} \mathrm{C}$ using molecular oxygen as stoichiometric oxidant. Catalysts containing $1 \mathrm{wt} \% \mathrm{Au}$ supported on $\mathrm{TiO}_{2}, \mathrm{Al}_{2} \mathrm{O}_{3}$, and $\mathrm{ZnO}$ were examined for ethanol oxidation. The results showed that $\mathrm{ZnO}$ and $\mathrm{TiO}_{2}$ gave higher initial activities as supports for gold in ethanol oxidation, followed by $\mathrm{Al}_{2} \mathrm{O}_{3}$. Ethanol conversions of $>90 \%$ and selectivities to acetic acid of $>95 \%$ were achieved when using $\mathrm{ZnO}$ and $\mathrm{TiO}_{2}$ as supports under conditions where a slight oxygen deficiency was used. With a slight excess of oxygen present initially, ethanol conversions of $99.4 \%$, and a selectivity to acetic acid selectivity of $99.8 \%$ could be achieved. Gold leaching seemed to be very apparent with alumina as support and also, after continued use with titania-based catalysts. The use of higher initial concentrations of ethanol (range studied 5 - 40 mass $\%$ ethanol in water) led to higher ethyl acetate selectivities. High acetic acid selectivities were seen for relatively low (5-10 mass\%) initial ethanol concentrations.
\end{abstract}

\footnotetext{
Molecular Sciences Institute, School of Chemistry, University of the Witwatersrand, Private Bag 3, PO Wits, 2050. South Africa 2 Advanced Materials Division, Mintek, Private Bag X 3015, Randburg, 2125, South Africa

* For correspondence: michael.scurrel/@wits.ac.za $\mathrm{Tel}+27117176716$, Fax +2711 7176749
}

Keywords: gold, ethanol, bioethanol, acetic acid, oxidation, catalysis

\section{Introduction}

The cost of various fuels and chemicals is largely governed by the cost of the raw material and the efficiency of the processes involved. Oil resources are becoming far more expensive compared with biomass resources. It is potentially possible to convert biomass (e.g. bioethanol) to essentially all high-value commodity chemicals and fuels currently available from fossil fuel resources. There could be additional advantages in using biomass as a feedstock compared with fossil resources, for example, in a chemical industry the introduction of oxygen functionalities into hydrocarbons can be rather difficult, whereas many products derived from biomass already contain some oxygen [1]. However, this entails an increase in production costs for some of the non-oxygen-containing products, such as gasoline [2]. It is possible to produce some chemicals more easily and in fewer steps from biomass than from fossil resources [3]. The use of biomass as a sustainable resource for production of fuels and commodity chemicals should offer an improved security in supply, since biomass can be grown in most parts of the world. Bioethanol could be one of the examples of biomass that can be used in future as a feedstock in this context.

Since the pioneering discovery by Haruta and his co-workers that gold nanoparticles can catalyze CO oxidation effectively at low temperatures [4] there has been a tremendous interest in gold-catalyzed oxidation reactions $[5,6]$. In particular, the oxidation of alcohols has received significant attention, and rightfully so since the reaction satisfies most of the demands for a modern, sustainable, and "green" reaction [7]. The selective oxidation of alcohols to carboxylic acids is a fundamental transformation in organic chemistry as these compounds are widely used as intermediates for fine chemicals [7-9]. The high activity of supported gold nanoparticles allows the use of air as the stoichiometric oxidant, leading to a relatively high atom economy. Furthermore, the 
reaction has the potential to play an important role in the synthesis of both commodity and fine chemicals, where the use of a heterogeneous catalyst allows the isolation of product(s) by a simple separation [10]. This represents a major improvement in catalysis compared with "traditional" oxidation technologies already used in the chemical industry, many of which often rely on the use of stoichiometric amounts of heavy metals. Traditional inorganic oxidants, such as permanganate and dichromate, are toxic and produce a large amount of waste. The separation and disposal of this waste material increases chemical production costs. Therefore, from both economic and environmental viewpoints, there is an urgent need for greener and more efficient methods that will replace these traditional toxic oxidants with clean oxidants, such as $\mathrm{O}_{2}$, preferably with the use of separable and reusable catalysts. Many researchers have reported the use of molecular oxygen as an oxidant for alcohol oxidation using different catalysts [11-15] and a variety of solvents. Due to the volatile, flammable, and toxic nature of organic solvents [16-18], solvent free oxidation has become the most preferred method. The oxidation of many organic substrates leads to the production of many functionalized molecules that are of great commercial and synthetic importance.

Previous investigations on ethanol oxidation have focused on metals such as platinum [19, 20], ruthenium [21], palladium [22, 23] and molybdenum [24]. An intense interest in gold catalysis followed the seminal work of Haruta et al [25] and Hutchings et al [26] in CO oxidation and acetylene hydrochlorination respectively. Recently, it has been found that Au supported catalysts are selective and stable for the oxidation of various alcohols to their corresponding aldehydes, acids and ketones [27-29]. In this emerging field of "green" oxidation chemistry, catalysts comprising gold nanoparticles have attained a very prominent role [30].

In this study, we have mainly investigated the catalytic behavior of $1 \mathrm{wt} \%$ Au catalysts supported on $\mathrm{ZnO}$ or $\mathrm{Al}_{2} \mathrm{O}_{3}$ or $\mathrm{TiO}_{2}$. The behavior of gold/titania in ethanol oxidation was previously reported [27]. We have also investigated the catalyst lifetime of $\mathrm{Au} / \mathrm{TiO}_{2}$ in ethanol oxidation reaction, as it has not been reported before, and have also noted the gold leaching tendencies of some of these catalysts.

\section{Experimental}

All gold catalysts were supplied by Mintek (AuTek catalysts) and contained 1 mass\% gold. Oxidation experiments were conducted in a $75 \mathrm{ml}$ stainless steel autoclave reactor. The autoclave was charged with $10 \mathrm{ml}$ of aqueous ethanol of desired concentration ranging from $5-40 \mathrm{wt} \%$ of ethanol in water, and 0.15 $\mathrm{g}$ of catalyst. It was then closed and charged with technical air $\left(80 \mathrm{vol} \% \mathrm{~N}_{2}, 20 \mathrm{vol} \% \mathrm{O}_{2}\right)$ at a required pressure of 48 bar. The autoclave was then heated to the desired reaction temperature of $150^{\circ} \mathrm{C}$ under stirring conditions for a required reaction time (5-25 h). The stirring rate was kept constant at $400 \mathrm{rpm}$ for all reactions. After each reaction, the autoclave was cooled with an ice-water mixture to a temperature below $5^{\circ} \mathrm{C}$, to help ensure that the volatile products such as acetaldehyde did not escape before analysis. At the end of each run, the liquid reaction mixture was separated from the catalyst by centrifuge, and then analyzed using a gas chromatograph equipped with a Carbowax column ( $2 \mathrm{~m} \times 2 \mathrm{~mm}$ I.D. glass $)$ and FID detector. The gas was also collected for analysis using a gas chromatograph equipped with a TCD detector. For all work reported here, the liquid samples obtained comprised a single-phase.

\section{Results and Discussion}

\section{Catalyst activity tests}

Figure 1 shows results for the catalytic activity runs using the various gold catalysts using molecular oxygen as the oxidant. Initially, ethanol concentrations of $5 \%$ in water were used. Gold on titanium oxide or zinc oxides showed a high initial activity towards ethanol oxidation. Ethanol conversions of $>90 \%$ was achieved (but a slight oxygen deficiency was used in these runs) After $10 \mathrm{~h}$ about $80 \%$ of ethanol conversion was observed, a result similar to that reported by Christensen et al where $1 \% \mathrm{Au} / \mathrm{MgAl}_{2} \mathrm{O}_{4}$ was used as a catalyst [27]. An ethanol conversion of $93 \%$ was observed when $1 \% \mathrm{Au} / \mathrm{TiO}_{2}$ was used in reactions conducted for longer than $20 \mathrm{~h}$ (slight oxygen deficiency used). When a slight excess oxygen was used, an ethanol conversion of $99.4 \%$, with an acetic acid selectivity of 99.8\% ( 100\%) were achieved. The data confirm that even when working with a deficiency of oxygen, no significant dehydrogenation is seen under our conditions. The activities seen when using $1 \% \mathrm{Au} / \mathrm{ZnO}$ and $1 \% \mathrm{Au} /$ $\mathrm{TiO}_{2}$ were similar. The mass balance was calculated to be close to $99-101 \%$ (all reactions were operated in a sealed batch reactor system). 


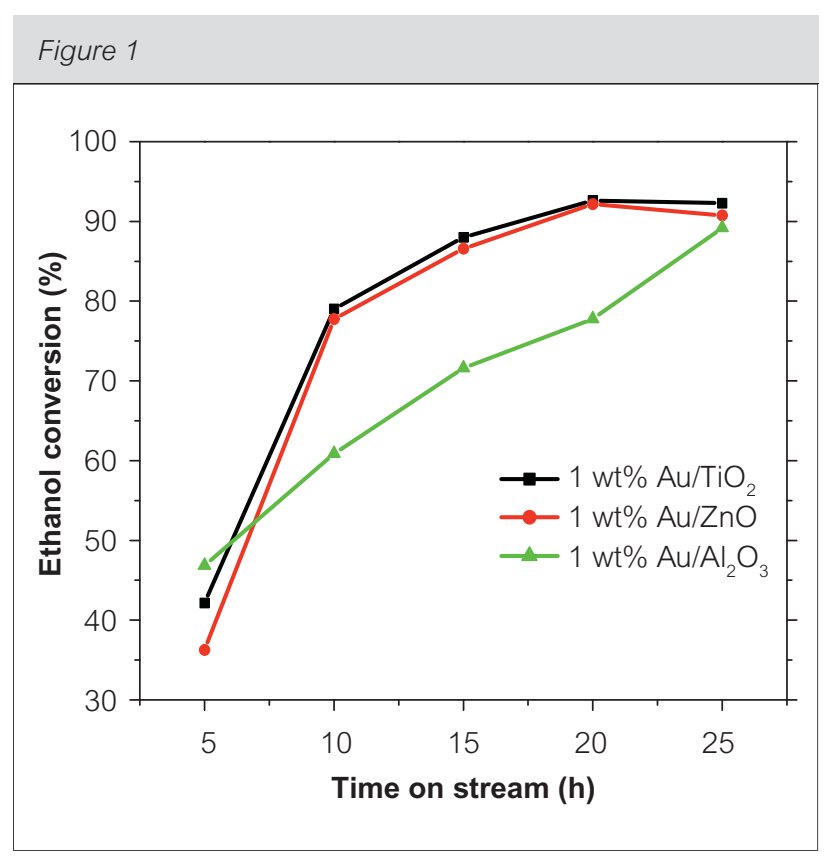

Conversion of ethanol as function of time using gold supported catalysts

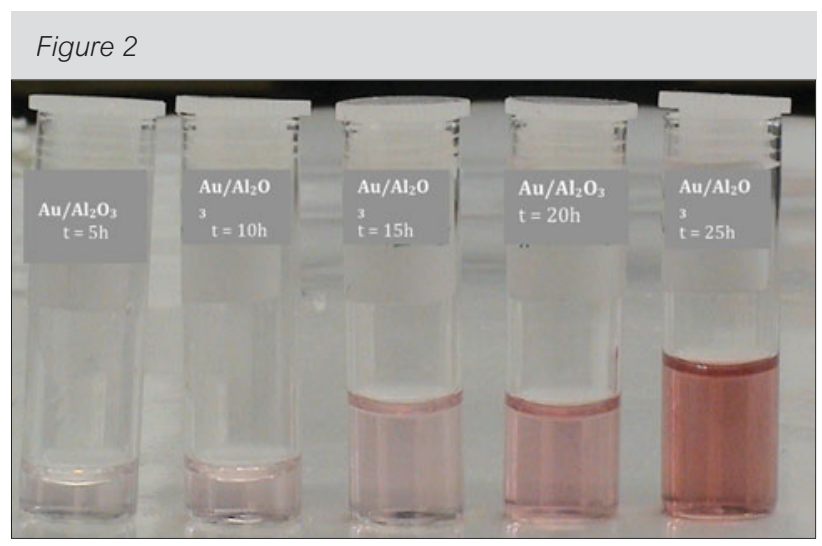

Gold leaching on $\mathrm{AuIAI}_{2} \mathrm{O}_{3}$ with increasing reaction time from left to right

The conversion-time trend observed when gold was supported on alumina $\left(\mathrm{Au} / \mathrm{Al}_{2} \mathrm{O}_{3}\right)$ was slightly different. Ethanol conversion increased almost linearly with increasing reaction time as shown in Figure 1. The conversion of ethanol observed was lower compared with that found with the other catalysts when reaction time was $<25 \mathrm{~h}$, but after 25 $\mathrm{h}$ the conversions achieved were essentially identical for all three catalyst types. However, gold leaching was clearly seen with the gold/alumina catalyst as shown in Figure 2.

Figure 3 shows the selectivity to acetic acid as a function of time. Au supported on titania or zinc oxide showed higher selectivities of $>95 \%$ to acetic acid, while that seen with $\mathrm{Au} / \mathrm{Al}_{2} \mathrm{O}_{3}$ was slightly below that level. It is possible that the lower selectivity seen in the case of the alumina support is connected with the more marked tendency of gold to leach from this catalyst, but further work is required in order to clarify this.

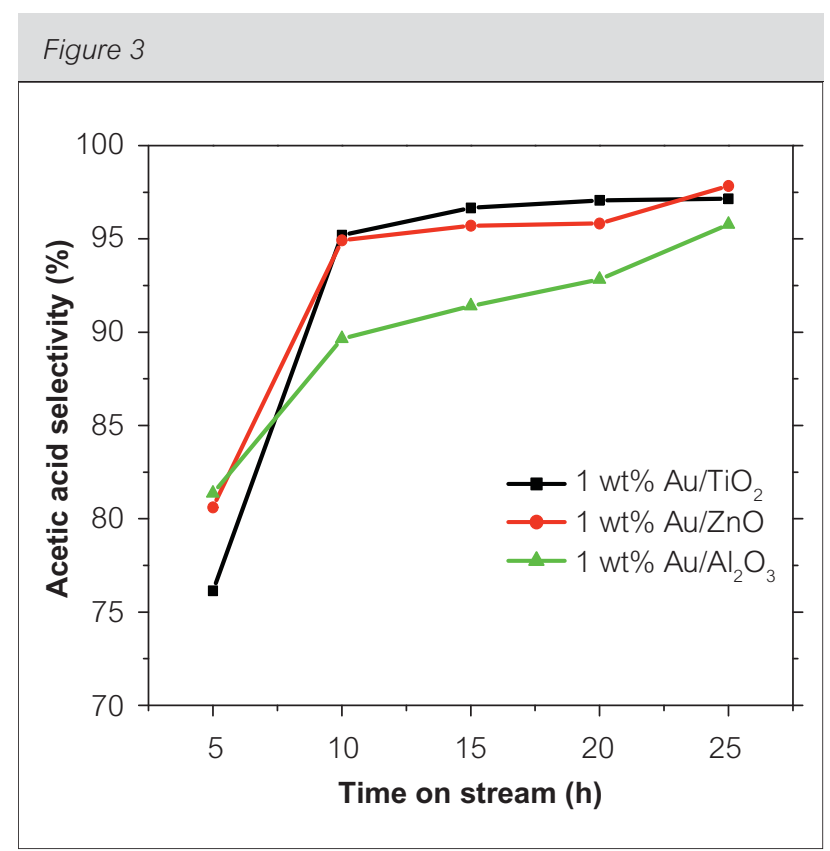

Selectivity of $1 \mathrm{wt} \%$ Au catalysts to acetic acid as function of time

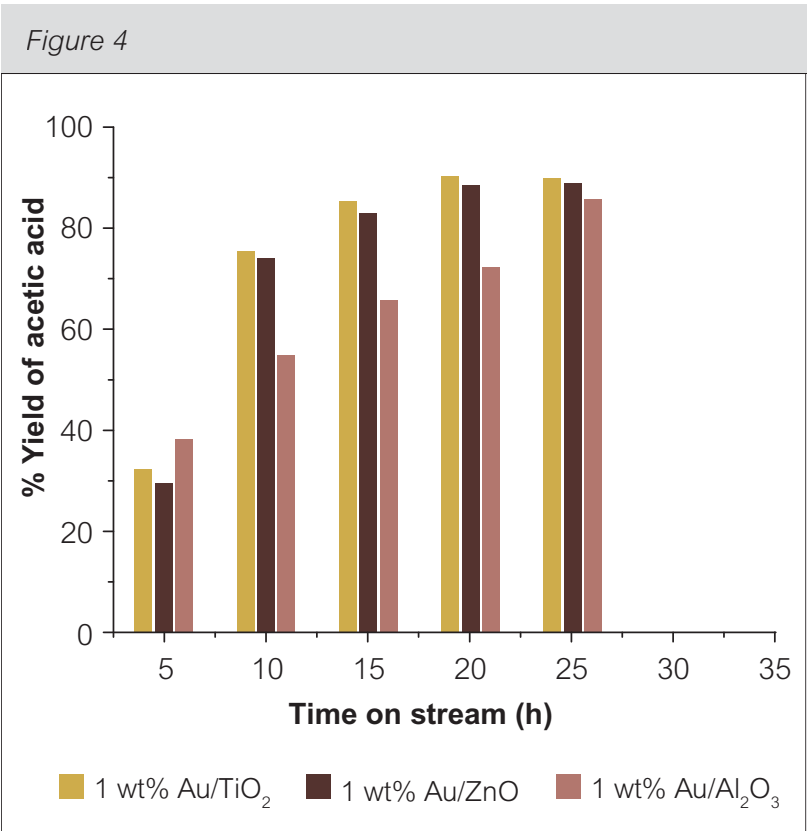

Yield of acetic acid as function of time 

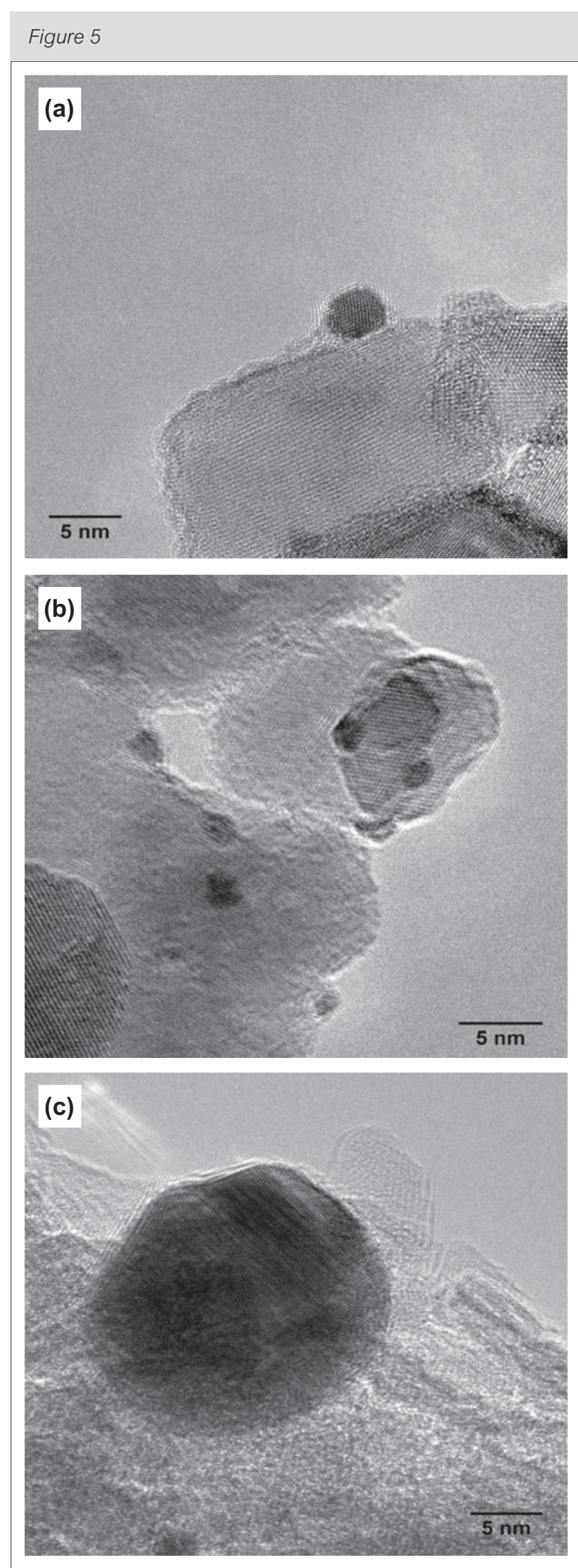

HRTEM images of (a) Au/TiO ${ }_{2}$, (b) $\mathrm{AulZnO}$ and (c) $\mathrm{AulAl}_{2} \mathrm{O}_{3}$
Figure 4 shows the yield of acetic acid obtained from three catalysts used in ethanol oxidation. Au supported on zinc oxide or titanium oxide showed higher selectivity to acetic acid. After $20 \mathrm{~h}$, a yield of about $90 \%$ acetic acid was achieved.

Figure 5 shows TEM images of the catalysts used in ethanol oxidation. TEM images were used to confirm the presence of gold in all three catalysts, and gold particle size distributions were calculated. The gold particle size distributions seen with $\mathrm{Au} /$ $\mathrm{TiO}_{2}$ and $\mathrm{Au} / \mathrm{ZnO}$ were very narrow with most of the particles being in the range of 2 to $3 \mathrm{~nm}$. The Au/ $\mathrm{Al}_{2} \mathrm{O}_{3}$ showed gold particle sizes $>10 \mathrm{~nm}$ and this might have contributed to the lower initial activity of this catalyst.

\section{Effect of ethanol concentration}

Figure 6 shows the effect of (initial) ethanol concentration on the oxidation reaction for the gold/zinc oxide catalyst. The mass of ethanol was kept constant for all reactions while varying the concentration from 5-40 wt\%. The conversion of ethanol was observed to decrease with increasing ethanol concentration at a given time. As the (initial) concentration of ethanol increased, it was noticed the rate of conversion is affected (lower rate), but the detailed reasons for the observed behavior must be sought in further studies of the kinetics, including changes in, for example, oxygen

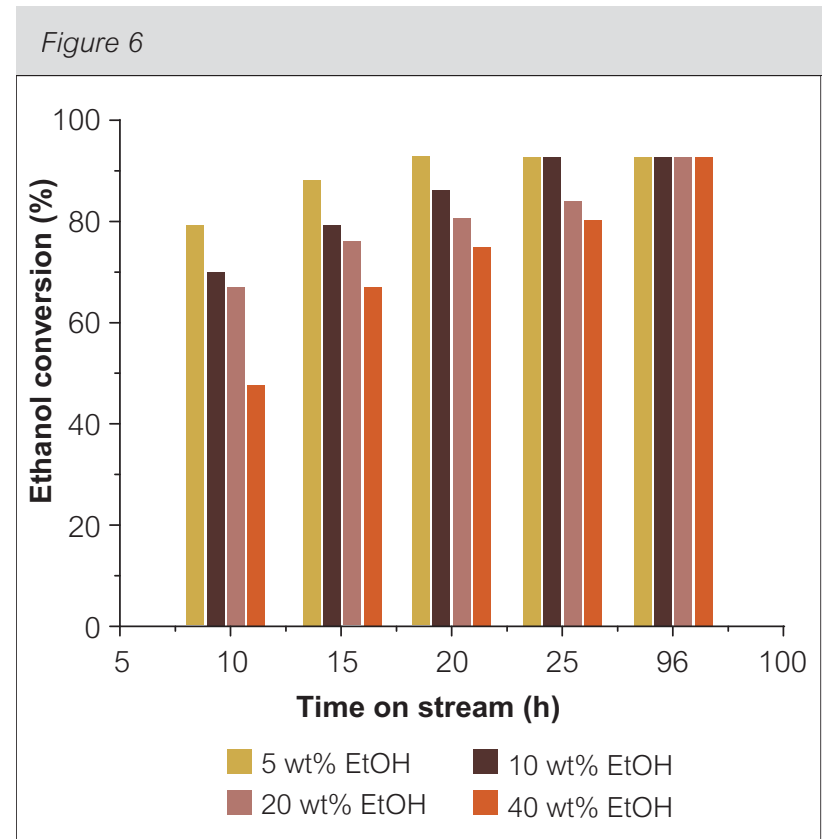

Effect of increasing ethanol concentration on oxidation of ethanol using $1 \mathrm{wt} \%$ AulZnO 


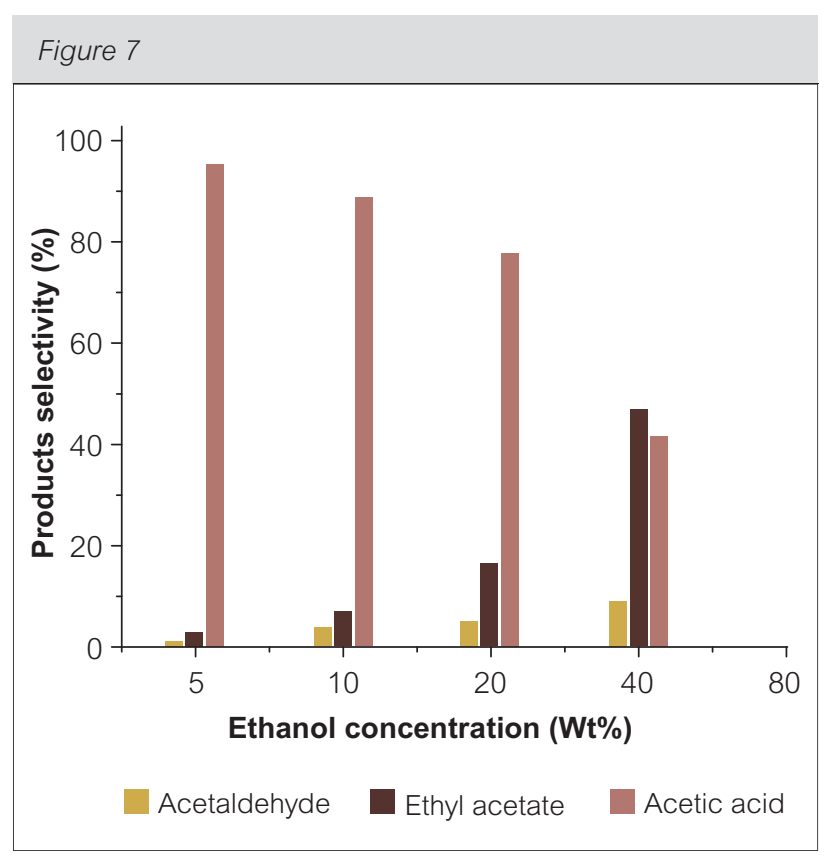

Product selectivity at different ethanol concentration; reactions were run for $20 \mathrm{~h}$

concentration in the liquid as a function of liquidphase composition. Ethanol conversion of $93 \%$ was achieved in all reactions with different initial ethanol concentration after the reactions had been run for 4 days (i.e. $96 \mathrm{~h}$ ).

The selectivities to the various products obtained as a function of different initial ethanol concentration are depicted in Figure 7. At higher initial ethanol concentrations, the production of ethyl acetate becomes more apparent, as was reported by Christiansen et al [31]. At 40 mass\% initial ethanol concentration, ethyl acetate is the major product after 20h.

Figure 8 shows a high yield of ethyl acetate at 40 wt $\%$ ethanol, compared with other two products. The yield of acetic acid decreased from 75 to 19\% with increasing initial ethanol concentration in the range $5-40 \mathrm{wt} \%$ respectively. It is crucial to work at low initial concentrations of ethanol (such as those encountered in typical fermentation conditions) in order to achieve high yields of acetic acid.

\section{Catalyst lifetime}

There are many factors that contribute to the deactivation of catalyst in aqueous phase oxidation reactions. The metal catalysts could be deactivated by blockage of the surface by strongly adsorbed molecules in the form of either impurities or reaction

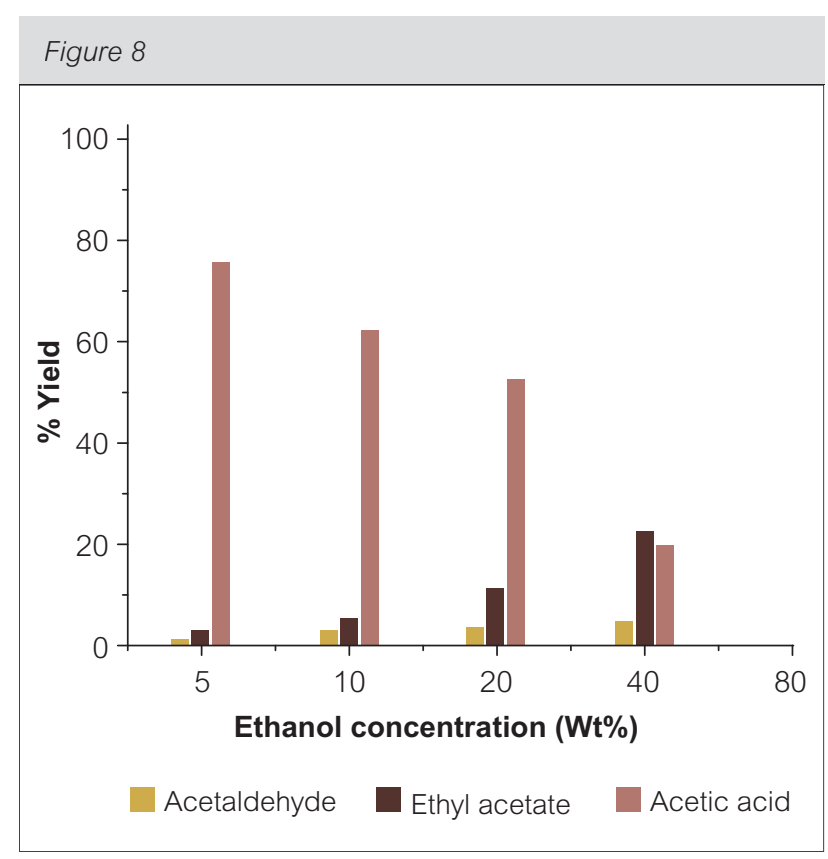

Yields at high ethanol concentration; reactions were run for $10 \mathrm{~h}$

products remaining adsorbed on the surface, or by adsorbed oxygen. Such catalysts can also exhibit deactivation through the leaching of metal into the liquid-phase.

Figure 9 shows the stability of $1 \mathrm{wt} \% \mathrm{Au} / \mathrm{TiO}_{2}$ in ethanol oxidation under the conditions described in the experimental section of this work. The catalyst was re-used in ethanol oxidation in order to assess the stability in the presence of an aqueous phase. At the end of each run the resulting solution was separated by centrifuge and the products were analyzed using gas chromatography and the remaining catalyst was washed with distilled water, air dried, then transferred to the next run. The conversion of ethanol in first and second reaction cycle was 93\%, but in a third cycle the activity of the catalyst dropped almost threefold to about $29 \%$, and this was attributed to the leaching of gold which was observed in a third reaction cycle. The study revealed that the catalyst can only be used twice, and still maintain higher ethanol conversion of $93 \%$. Throughout this work, hot filtration tests showed that the filtrate was unable to catalyze the oxidation reaction and these catalysts therefore function in a truly heterogeneous manner. It is not yet clear why the reduced activity was observed only in the third cycle. 


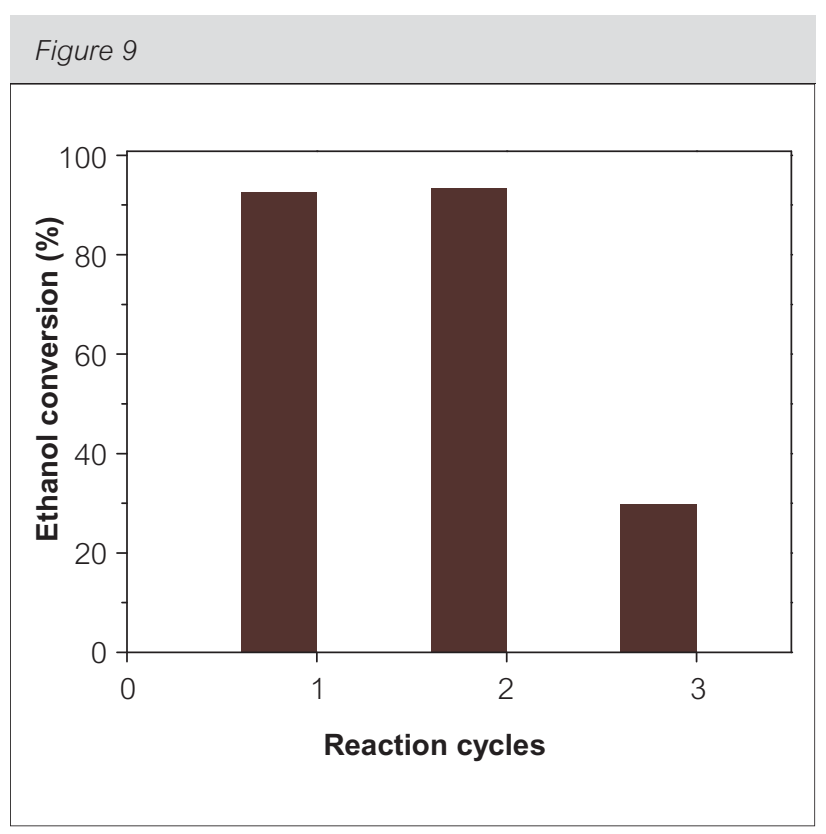

Stability of 1 wt\% AulTiO ${ }_{2}$ on ethanol oxidation

\section{Conclusions}

Results of this work show that acetic acid can be produced from ethanol by a selective oxidative process over supported gold catalysts. Ethanol conversion of $>90 \%$ and a selectivity to acetic acid of $>95 \%$ to acetic acid was achieved when using $\mathrm{ZnO}$ as support for $\mathrm{Au}$, under conditions where a slight oxygen deficiency was employed. When sufficient oxygen (slight excess) was used in a batch reactor an ethanol conversion of $99.4 \%$ and an acetic acid selectivity of $99.8 \%$ was achieved. Alumina supported gold showed a somewhat poorer performance in ethanol oxidation, and pronounced leaching of gold into solution. Comparisons can now also be made with the data obtained by others using gold on $\mathrm{TiO}_{2}$ and $\mathrm{MgAl}_{2} \mathrm{O}_{4}$ as supports. We have also found that the stability of the gold/titania system in our hands is limited, with the catalyst exhibiting a marked reduction in activity during the third cycle of use under our conditions. The use of higher initial ethanol concentrations favour the production of ethyl acetate rather than acetic acid. These preliminary studies suggest some characteristics of supported gold for ethanol oxidation. More systematic work is required in order to assess whether such catalysts can be used for the economic conversion of bioethanol to acetic acid, for example. In particular we note that there is a need for optimization work (catalyst design, selection of process conditions, catalyst durability).

\section{Acknowledgements}

Mintek and Project AuTek (Mintek and AnglogoldAshanti) are gratefully acknowledged for financial support and for the provision of catalysts (AURO/ite ${ }^{\mathrm{TM}}$ catalysts).

\section{References}

1 B.E. Dale, J. Chem. Technol. Biotechnol. 2003, 78, 1093

2 J. Rass-Hansen, H. Falsig, B. Jørgensen, C.H. Christensen, J. Chem. Technol. Biotechnol. 2007, 82, 329

3 N. Kosaric, Z. Duvnjak, A. Farkas, H. Sahm, S. BringerMeyer, O. et al Goebel, Ethanol in Ullmann's Encyclopedia of Industrial Chemistry (7th edn). Wiley, Chichester, 2006

4 M. Haruta, T. Kobayashi, H. Sano, N. Yamada, Chem. Lett. 1987, 16, 405

5 A.S.K. Hashmi, G.J. Hutchings, Angew. Chem. Int. Ed. 2006, 45, 7896

6 P.P. Edwards, J.M. Thomas, Angew. Chem. Int. Ed. 2007, 46, 5480

7 T. Mallat, A. Baiker, Chem. Rev. 2004, 104, 3037

8 J. Muzart, Tetrahedron, 2003, 59, 5789

9 J.S. Rafelt, J.H. Clark, Catal. Today, 2000, 57, 33

10 T. Ishida, M. Haruta, Angew. Chem. Int. Ed. 2007, 46, 7154

11 Z. Opre, D. Ferri, F. Krumeich, T. Mallat, A. Baiker, J. Catal. 2006, 241, 287

12 D.V. Bavykin, A.A. Lapkin, S.T. Kolaczkowski, P.K. Plucinski, Appl. Catal. A. 2005, 288, 175

13 K. Mori, T. Hara, T. Mizugaki, K. Ebitani, K. Kaneda, J. Am. Chem. Soc. 2004, 126, 10657

14 H. Ji, J. Song, B. He, Y. Qian, React. Kinet. Catal. Lett. 2004, 82, 97

15 V.D. Makwana, Y.C. Son, A.R. Howell, S.L. Suib, J. Catal. 2002, 210, 46

16 H.J. Kluytmans, A.P. Markusse, B.F. Kuster, G.B. Marin, J.C. Schouten, Catal. Today, 2000, 57, 143

17 V.R. Gangwal, J. van der Schaaf, B.M. Kuster, J.C. Schouten, J. Catal. 2005, 232, 432

18 G.J. Hutchings, S. Carrettin, P. Landon, J.K. Edwards, D. Enache, D.W. Knight, Y. Xu, A.F. Carley, Top. Catal. 2006, 38, 223

19 A.F. Lee, D.E. Gawthrope, N.J. Hart, K. Wilson, Surf. Sci. 2004, 548, 200

20 M.K. Rajumon, M.W. Roberts, F. Wang, P.B. Wells, J. Chem. Soc. Faraday Trans. 1998, 94, 3699

21 E. Vessellie, A. Baraldi, G. Comelli, S. Lizzit, R. Rosei, Chemphyschem. 2004, 5, 1113

22 M. Bowker, R.P. Holroyd, R.G. Sharpe, J.S. Corneille, S.M. Francis, D.W. Goodman, Surf. Sci. 1997, 370, 113

23 J.L. Davis, M.A. Barteau, Surf. Sci. 1988, 197, 123

24 D.A. Chen, C.M. Friend, J. Am. Chem. Soc. 1998, 120, 5017

25 M. Haruta, T. Kobayashi, H. Sano, N. Yamada, Chem Lett. 1987, 405 
26 G.J. Hutchings, J. Catal. 1985, 96, 292

27 C.H. Christensen, B. Jørgensen, J. Rass-Hansen,

K. Egeblad, R. Madsen, S.K. Klitgaard, S.M. Hansen,

M.R. Hansen, H.C. Andersen, A. Riisager, Angew. Chem., Int. Ed. 2006, 45, 4648

28 D.L. Enache, J.K. Edwards, P. Landon, B. Solsona-Espriu, A.F. Carley, A.A. Herzing, M. Watanable, C.J. Kiely,

D.W. Knight, G.J. Hutchings, Science 2006, 311, 362

29. S. Biella, M. Rossi, Chem. Commun. 2003, 378

30 A.S.K. Hashmi, G.J. Hutchings, Angew. Chem. Int. Ed. 2006,

45, 7896

31 B. Jørgensen, S.E. Christiansen, M.L.D. Thomsen,

C.H. Christensen, Appl. Catal. A: Gen. 2007, 251, 332 